\title{
Dynamic Modeling of a Boring Bar Using Theoretical and Experimental Engineering Methods Part 1: Distributed-Parameter System Modeling and Experimental Modal Analysis
}

\author{
Tatiana Smirnova \\ Department of Signal Processing, Blekinge Institute of Technology, PO Box 520, SE-37225 Ronneby, Sweden
}

\author{
Henrik Åkesson \\ Department of Signal Processing, Blekinge Institute of Technology, PO Box 520, SE-37225 Ronneby, Sweden \\ Acticut International AB, Gjuterivägen 7, 31132 Falkenberg, Sweden
}

\author{
Lars Håkansson \\ Department of Signal Processing, Blekinge Institute of Technology, PO Box 520, SE-37225 Ronneby, Sweden
}

\begin{abstract}
Boring bar vibration is a common problem during internal turning operations and is a major problem for the manufacturing industry. High levels of boring bar vibration generally occur at frequencies related to the first two fundamental bending modes of a boring bar. This is the first of two companion papers that summarize the theoretical and experimental work carried out concerning modeling of dynamic properties of boring bars. This paper introduces the Timoshenko beam theory for the modeling of clamped boring bars. Also, the traditional Euler-Bernoulli beam theory is applied. These continuous system methods have been utilized to produce fixedfree beam models of the clamped boring bar. In order to improve accuracy of dynamic models of clamped boring bars, the modeling of the boring bar clamping is addressed by means of multi-span beam models with pinned boundary conditions. The derived boring bar models have also been compared with results obtained by means of experimental modal analysis, conducted on the actual boring bar clamped in a lathe. The multi-span beam boring bar models display higher correlation with experimental modal analysis results as compared to fixed-free beam models. For the fixed-free beams the Timoshenko model results in the highest correlation with the experimental results. On the other hand, the interval in frequency and the orientation of the two fundamental modes demonstrate differences, particularly between the continuous system models and the experimental results.
\end{abstract}

\section{INTRODUCTION}

The internal turning operation is known to be one of the most troublesome with regard to vibrations in metal cutting. During such an operation a boring bar tool cuts deep, precise cavities into a workpiece material. However, due to geometric dimensions that a boring bar generally is required to have in order to perform the boring operation (i.e., large length to diameter ratio), the bar is easily subjected to vibrations. The boring bar vibrations are generally dominated by one of the two fundamental bending modes of the bar, and the vibration level is usually greatest in the cutting speed direction. ${ }^{1,2}$ Boring bar vibrations commonly lead to a poor workpiece surface finish, reduced tool life, and severe acoustic noise levels, and have a negative impact on factors such as productivity and production costs.

A number of experimental and analytical studies have been carried out to study boring bar dynamics. Most research has usually been carried out on modeling of cutting dynamics and frequently concentrates on the prediction of stability limits. Based on a two-degrees-of freedom analytical model of a boring bar with two input forces, one proportional to the variation of chip thickness and the other proportional to the penetration velocity, Parker ${ }^{3}$ predicted stability limits of a slender boring bar in external longitudinal turning. ${ }^{3}$ From boring bar point receptance estimates, the modal constants of the model were determined. However, there was a wide range of cutting speeds resulting in extensive vibration in the cutting speed direction, which was not predicted by the model. Zhang et al. also modeled a boring bar as a system with two degrees-of-freedom, but in the form of a linear state-variable system model. ${ }^{4}$ As state variables, the displacements and velocities of the two first principal modes of the boring bar were used. The impulse response method was used to estimate natural frequencies and damping ratios for the two principal modes. Also, a dynamic cutting force model based on four cutting force components was included. Based on their analytical model, Zhang et al. predicted the limit width-of-cut for a boring bar in an external thread cutting process. They assert that a good agreement was found between the predicted and measured values of the limit width of cut . Pratt et al. developed among other things a model of cutting dynamics based on a two-degree-of-freedom system to predict the limit width of cut. ${ }^{5}$ They state that the predicted limit width of cut is in reasonable agreement with that observed experimentally. Rao et al. approximated a boring bar as a continuous system cantilever beam with "fixed-free" 